\title{
Two-dimensional electromagnetic solitons in a perpendicularly magnetized ferromagnetic slab
}

\author{
H. Leblond ${ }^{1}$ and M. Manna ${ }^{2}$ \\ ${ }^{1}$ Laboratoire POMA, CNRS-FRE 2988, Université d'Angers, 2 Boulevard Lavoisier 49045 Angers Cedex 1, France \\ ${ }^{2}$ Laboratoire de Physique Théorique et Astroparticules IN2P3/CNRS-UMR 5207, Université Montpellier II, \\ Place E. Bataillon, 34095 Montpellier Cedex 05, France
}

(Received 27 February 2009; revised manuscript received 2 June 2009; published 27 August 2009)

\begin{abstract}
We consider the nonlinear propagation of bulk polaritons in a magnetically saturated ferromagnetic thick film, the applied magnetic field being perpendicular to the film plane. A $(2+1)$-dimensional asymptotic model equation generalizing the sine-Gordon one is derived. Line-soliton solutions are exhibited, their stability condition is derived. When unstable, line solitons decay into stable two-dimensional lumps, which are studied by means of variational analysis.
\end{abstract}

DOI: $10.1103 /$ PhysRevB.80.064424

PACS number(s): 05.45.Yv, 41.20.Jb, 75.30.Ds

\section{INTRODUCTION}

Magnetic and electromagnetic waves in ferromagnetic media present a lot of remarkable features due to the highly nonlinear character of the coupling between magnetization and magnetic field. ${ }^{1}$

Regarding magnetostatic modes, envelope solitons have been observed ${ }^{2,3}$ and described theoretically. ${ }^{4,5}$ In the electromagnetic or polariton domain, envelope solitons have also been described theoretically. 6,7 Solitary waves, i.e., singleoscillation structures, have also been predicted. ${ }^{8-13}$ They can be stable in one, two, or three dimensions. However the size predicted theoretically for these structures exceeds the available samples. Henceforth, the experimental results restrict to the linear case, ${ }^{14,15}$ for which theoretical models have been developed with a high accuracy. ${ }^{16-18}$

Recently, one- ${ }^{19,20}$ and two- ${ }^{21,22}$ dimensional solitons have been evidenced by means of the short-wave approximation. The sample was assumed to be saturated by an external field belonging to the film plane and perpendicular to the propagation direction. Stable localized structures called lumps have been evidenced. Using parameters typical for yttrium iron garnet (YIG), their size has been found typically less than $0.2 \mathrm{~mm}$ in length and about $3-4 \mathrm{~mm}$ in width. ${ }^{23} \mathrm{~A}$ particular feature of the situation just mentioned is that the applied field breaks the symmetry and induces a transverse drift of the waves.

We analyze here the same process but with a field perpendicular to the plane of the thick film; we will see that lumps also exist, except that the symmetry is restored and the analysis simplifies.

\section{SOME APPROXIMATIONS}

\section{A. Basic equations and assumptions}

We consider a ferromagnetic thick film lying in the $x y$ plane, $x$ being the propagation direction. The film is magnetized to saturation by an external field $\mathbf{H}_{\text {ext }}=\left(0,0, H_{\text {ext }}\right)$ directed along $z$, see Fig. 1 . The evolution of the magnetic field $\mathbf{H}$ is governed by the Maxwell equations, which reduce to

$$
-\nabla(\nabla \cdot \mathbf{H})+\Delta \mathbf{H}=\frac{1}{c^{2}} \frac{\partial^{2}}{\partial t^{2}}(\mathbf{H}+\mathbf{M}),
$$

where $c=1 / \sqrt{\mu_{0} \widetilde{\varepsilon}}$ is the speed of light in the medium, with $\widetilde{\varepsilon}$ its scalar permittivity. For YIG we can take the relative permittivity of the medium as $\widetilde{\varepsilon} / \varepsilon_{0}=12$, then $c=8.66$ $\times 10^{7} \mathrm{~ms}^{-1}$. The magnetization density $\mathbf{M}$ obeys the Landau-Lifschitz equation

$$
\frac{\partial}{\partial t} \mathbf{M}=-\gamma \mu_{0} \mathbf{M} \wedge \mathbf{H}+\frac{\sigma}{M_{s}} \mathbf{M} \wedge(\mathbf{M} \wedge \mathbf{H}),
$$

where $\gamma$ is the gyromagnetic ratio, $\mu_{0}$ is the magnetic permeability in vacuum, $\sigma<0$ is the damping constant, and $M_{s}$ is the saturation magnetization. $\mathbf{H}$ in Eqs. (1) and (2) is the internal magnetic field, which is related to the external field $\mathbf{H}_{\text {ext }}$ through

$$
\mathbf{H}=\mathbf{H}_{\mathrm{ext}}-N \cdot \mathbf{M} .
$$

in which $N$ is the demagnetizing factor tensor. For a film lying long the $x y$ plane, $N$ is diagonal with $\left(N_{x}, N_{y}, N_{z}\right)$ $=(0,0,1)$. For a thick enough film and for volume modes, the finite thickness of the film and the continuity conditions at the film boundaries can be approximately accounted for by dropping the $z$ derivative, and making use of relation (3). We consider bulk polaritons with typical wavelengths ranging from 10 to $100 \mu \mathrm{m}$, in films with a typical thickness of 0.5 $\mathrm{mm}$. The wavelengths considered are large with regard to the exchange length and hence inhomogeneous exchange can be neglected. We also assume that the crystalline and surface anisotropy of the sample are negligible.

\section{B. Linear analysis}

In order to study the linear regime, we linearize Eqs. (1) and (2) about the steady state

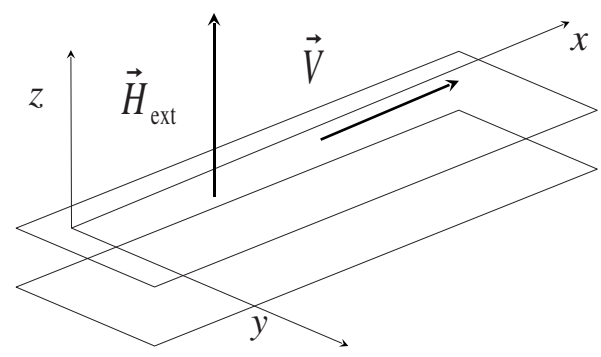

FIG. 1. The configuration considered. 


$$
\mathbf{M}_{0}=\left(0,0, M_{s}\right), \quad \mathbf{H}_{0}=\left[0,0,(\alpha-1) M_{s}\right],
$$

so that $\alpha$ measures the strength of the external field is units of $M_{s}$, as

$$
\alpha=\frac{H_{\mathrm{ext}}}{M_{s}} .
$$

The static fields $\mathbf{M}_{0}$ and $\mathbf{H}_{0}$ lie both along the $z$ coordinate so they are perpendicular to the $x y$ plane, which is the plane of the slab. Then we look for solutions propagating in the plane of the slab and proportional to $\exp i \theta$ with $\theta$ being a twodimensional phase given by $\theta=k_{x} x+k_{y} y-\omega t$, where $k_{x}$ and $k_{y}$ are the wave numbers in the $x, y$ directions and $\omega$ is the frequency. So, the magnetization density and magnetic field express as $\mathbf{M}=\mathbf{M}_{0}+\mathbf{M}_{1} e^{i \theta}$ and $\mathbf{H}=\mathbf{H}_{0}+\mathbf{H}_{1} e^{i \theta}$, where $\mathbf{M}_{1}$ $=\left(m_{x}, m_{y}, m_{z}\right)$ and $\mathbf{H}_{1}=\left(h_{x}, h_{y}, h_{z}\right)$ are small constant vectors to be determined. Neglecting the damping, Eqs. (1)-(3) reduce to $m_{z}=h_{z}=0$ and

$$
\left(\begin{array}{cccc}
\omega^{2} / c^{2} & 0 & \omega^{2} / c^{2}-k_{y}^{2} & k_{x} k_{y} \\
0 & \omega^{2} / c^{2} & k_{x} k_{y} & \omega^{2} / c^{2}-k_{x}^{2} \\
i \omega / c & (1-\alpha) / l_{0} & 0 & 1 / l_{0} \\
\alpha / l_{0} & i \omega / c & -1 / l_{0} & 0
\end{array}\right) \cdot\left(\begin{array}{c}
m_{x} \\
m_{y} \\
h_{x} \\
h_{y}
\end{array}\right)=0
$$

where we have set

$$
l_{0}=\frac{c}{\gamma \mu_{0} M_{s}} .
$$

The characteristic length $l_{0}$ is the wavelength of the "optical" wave at the frequency of the ferromagnetic resonance for an applied field equal to the saturation magnetization.

Then we obtain the dispersion relation

$$
\frac{\omega^{2}}{c^{2}}\left(\frac{\omega^{2}}{c^{2}}-k_{x}^{2}-k_{y}^{2}\right)-\frac{\alpha}{l_{0}^{2}}\left[\frac{\omega^{2}}{c^{2}}+(\alpha-1)\left(\frac{\omega^{2}}{c^{2}}-k_{x}^{2}-k_{y}^{2}\right)\right]=0 .
$$

The short-wave approximation is possible when the dispersion relation admits an expansion of the form ${ }^{24,25}$

$$
\omega=\frac{a_{0}}{\varepsilon}+a_{1} \varepsilon+a_{2} \varepsilon^{3}+a_{3} \varepsilon^{5} \cdots,
$$

where the small parameter $\varepsilon$ is related to the magnitude of the wavelength through $k_{x}=k_{x}^{0} / \varepsilon$, which corresponds to short waves. The direction of the wave propagation is assumed to be close to the $x$ axis, in such a way that the $y$ variable gives only account of a slow transverse deviation. Therefore $k_{y}$ is assumed to be very small with respect to $k_{x}$ and we write $k_{y}=k_{y}^{0}$, of order 0 with respect to $\varepsilon$. The reference values $k_{x}^{0}$ and $k_{y}^{0}$ have the same order of magnitude as $2 \pi / l_{0}$. If we consider typical values for $\mathrm{YIG}$, as $M_{s}\left(\right.$ or $\left.4 \pi M_{s}\right)=1800 \mathrm{Oe}$, $\gamma \mu_{0}=1.759 \times 10^{7} \mathrm{rad} \mathrm{s}^{-1} \mathrm{Oe}^{-1}$, and the above values of $c$, we get $l_{0} \simeq 2.73 \mathrm{~mm}$. In this case, $\varepsilon=0.01$ corresponds to a wavelength $2 \pi / k_{x}^{0} \sim \varepsilon l_{0} \sim 27 \mu \mathrm{m}$. Computation of the two first coefficients $a_{0}$ and $a_{1}$ gives

$$
a_{0}=c k_{x}^{0}, \quad a_{1}=\frac{c}{2 k_{x}^{0}}\left[\frac{\alpha}{l_{0}^{2}}+\left(k_{y}^{0}\right)^{2}\right],
$$

which proves that the short-wave approximation is possible. The propagation is mainly in the $x$ direction with a small deviation in the $y$ direction. The phase up to order $\varepsilon$ is thus

$$
\theta=\frac{1}{\varepsilon} k_{x}^{0}(x-t)+k_{y}^{0} y-\varepsilon b t,
$$

with $a_{0}$ and $a_{1}$ given by Eq. (10), which motivates the introduction of the scaled variables

$$
\zeta=\frac{1}{\varepsilon}(x-V t), \quad y=y, \quad \tau=\varepsilon t .
$$

The variable $\zeta$ describes the shape of the wave propagating at speed $V$, it assumes a short wavelength about $\varepsilon$. The slow time variable $\tau$ accounts for the propagation during very long time on distances very large with regard to the wavelength. The transverse variable $y$ has an intermediate scale, as in Kadomtsev-Petviashvili (KP)-type expansions. ${ }^{26,27}$

\section{A TWO-DIMENSIONAL GENERALIZATION OF THE SINE-GORDON EQUATION}

\section{A. Multiple scale approach}

Let us turn to the nonlinear aspect. Equation (12) allows us to introduce rescaled space and time operators, as

$$
\frac{\partial}{\partial x}=\frac{1}{\varepsilon} \frac{\partial}{\partial \zeta}, \quad \frac{\partial}{\partial y}=\frac{\partial}{\partial y}, \quad \frac{\partial}{\partial t}=\frac{-V}{\varepsilon} \frac{\partial}{\partial \zeta}+\varepsilon \frac{\partial}{\partial \tau} .
$$

The fields $\mathbf{M}$ and $\mathbf{H}$ are expanded in power series of $\varepsilon$, as

$$
\begin{gathered}
\mathbf{M}=\mathbf{M}_{0}+\varepsilon \mathbf{M}_{1}+\varepsilon^{2} \mathbf{M}_{2}+\ldots, \\
\mathbf{H}=\mathbf{H}_{0}+\varepsilon \mathbf{H}_{1}+\varepsilon^{2} \mathbf{H}_{2}+\ldots,
\end{gathered}
$$

where $\mathbf{M}_{0}, \mathbf{H}_{0}, \mathbf{M}_{1}, \mathbf{H}_{1}, \ldots$ are functions of $(\zeta, y, \tau)$. The perturbation of the magnetization and magnetic field is assumed to be localized so that $\mathbf{M}$ and $\mathbf{H}$ tend at infinity to the steady state defined by the external field $\mathbf{H}_{\text {ext }}$ perpendicular to the film and the demagnetizing field, cf. Eq. (3). Hence the $\mathbf{M}_{j}$ and $\mathbf{H}_{j}$ vanish at infinity, except $\mathbf{M}_{0}$ and $\mathbf{H}_{0}$, which tend to $\left(0,0, M_{s}\right)$ and $\left[0,0,(\alpha-1) M_{s}\right]$, respectively.

As written above, the reference length is $l_{0}$, typically about $3 \mathrm{~mm}$. Hence the scaling Eq. (12) assumes that $y$ varies over a range of a few $l_{0}$ while the length of the wave is about $l_{0} / \varepsilon \sim 30 \mu \mathrm{m}$ if we assume $\varepsilon=0.01$. In order that the confinement in the $z$ direction can be accounted for by merely dropping the $z$ variable and using the demagnetizing field in the boundary condition at infinity according to Eq. (3), $z$ must have an order of magnitude intermediary between $x$ and $y$, say $l_{0} / \sqrt{\varepsilon}$ which is about $0.3 \mathrm{~mm}$ with the above values. Hence the theory is expected to be valid for films with thicknesses in this range.

Further, damping is weak. In YIG films, the dimensionless damping parameter $\tilde{\sigma}=\sigma / \mu_{0} \gamma$ can be as small as $10^{-4}$ (see Ref. 28). For $\varepsilon \simeq 0.01$, it is hence of order $\varepsilon^{2}$. We will show below that under this assumption, the effect of the damping 
can be completely neglected within the short-wave approximation. In order to be able both to justify this statement and to derive an equation which takes into account a stronger damping, we set $\tilde{\sigma}=\varepsilon \bar{\sigma}$. The case of a YIG film with low losses will be recovered by setting $\bar{\sigma}$ to zero while a nonzero value of $\bar{\sigma}$ will account for higher losses.

Expansions (14), etc..., and scaling (12) are substituted into the Eqs. (1) and (2) and solved order by order. At leading order $1 / \varepsilon^{2}$ in the Maxwell equation (1) and $1 / \varepsilon$ in the Landau-Lifschitz one (2) it is found that the velocity $V=c$, i.e., the speed of light in the medium, $\mathbf{M}_{0}$ is a constant and $H_{0}^{x}$ is zero while $H_{0}^{y}$ and $H_{0}^{z}$ remain free. At order $1 / \varepsilon$ in Eq. (1) and $\varepsilon^{0}$ in Eq. (2), we find that

$$
\mathbf{M}_{1}=\left(\begin{array}{c}
\frac{-1}{l_{0}} \int_{-\infty}^{\zeta} H_{0}^{y} d \zeta^{\prime} \\
0 \\
0
\end{array}\right) \text {, }
$$

in which the characteristic length $l_{0}$ is defined as above as $l_{0}=c /\left(\mu_{0} \gamma M_{s}\right)$ and

$$
H_{1}^{x}=\frac{1}{l_{0}} \int_{-\infty}^{\zeta} H_{0}^{y} d \zeta^{\prime}-\int_{-\infty}^{\zeta} \frac{\partial H_{0}^{y}}{\partial y} d \zeta^{\prime}
$$

At order $\varepsilon^{0}$ in Eq. (1) and $\varepsilon$ in Eq. (2), after eliminating $\mathbf{H}_{2}$ and $\mathbf{M}_{2}$, we obtain

$$
\begin{gathered}
\frac{\partial^{2} H_{1}^{x}}{\partial \zeta \partial y}+\frac{1}{l_{0}} \frac{\partial}{\partial \zeta}\left[H_{1}^{x}-\frac{M_{1}^{x} H_{0}^{z}}{M_{s}}+\bar{\sigma} H_{0}^{y}\right]-\frac{2}{c} \frac{\partial^{2} H_{0}^{y}}{\partial \zeta \partial \tau}=0, \\
\frac{\partial^{2} H_{0}^{z}}{\partial y^{2}}-\frac{1}{l_{0} M_{s}} \frac{\partial}{\partial \zeta}\left[M_{1}^{x} H_{0}^{y}\right]+\frac{2}{c} \frac{\partial^{2} H_{0}^{z}}{\partial \zeta \partial \tau}=0 .
\end{gathered}
$$

Then $M_{1}^{x}$ and $H_{1}^{x}$ given by Eqs. (16) and (17) are substituted into Eqs. (18) and (19) to yield the following system of evolution equations for $H_{0}^{y}$ and $H_{0}^{z}$ :

$$
\frac{2}{c} \frac{\partial^{2} H_{0}^{y}}{\partial \zeta \partial \tau}=\frac{H_{0}^{y}}{l_{0}^{2}}-\frac{\partial^{2} H_{0}^{y}}{\partial y^{2}}+\frac{1}{l_{0}^{2} M_{s}} \frac{\partial}{\partial \zeta}\left[H_{0}^{z} \int_{-\infty}^{\zeta} H_{0}^{y} d \zeta^{\prime}\right]+\frac{\bar{\sigma}}{l_{0}} \frac{\partial H_{0}^{y}}{\partial \zeta}
$$

$$
\frac{2}{c} \frac{\partial^{2} H_{0}^{z}}{\partial \zeta \partial \tau}=-\frac{\partial^{2} H_{0}^{z}}{\partial y^{2}}-\frac{1}{l_{0}^{2} M_{s}} \frac{\partial}{\partial \zeta}\left[H_{0}^{y} \int_{-\infty}^{\zeta} H_{0}^{y} d \zeta^{\prime}\right]
$$

\section{B. Asymptotic model}

System (20)-(21) reduces to

$$
\begin{gathered}
C_{X T}=-B B_{X}+C_{Y Y}, \\
B_{X T}=B C_{X}+B_{Y Y}-s B_{X},
\end{gathered}
$$

where we use the dimensionless field and variables

$$
X=\frac{-\zeta}{2 l_{0}}, \quad Y=\frac{y}{l_{0}}, \quad T=\frac{c \tau}{l_{0}},
$$

$$
H_{0}^{y}=-M_{s} B_{X}, \quad H_{0}^{z}=-M_{s}\left(1+C_{X}\right), \quad s=\frac{-\bar{\sigma}}{2},
$$

and the subscript denotes partial derivative. The system (22)(23) differs from the one derived in Refs. 20-22 in its linear terms. The transverse drift, i.e., the terms involving $B_{Y}$ and $C_{Y}$, is absent. This fact in turn has important consequences in the nonlinear dynamics of the magnetic field $\vec{H}_{0}$.

The scaling of the variables is the same, the expression of fields is comparable: the $y$ and $z$ components are inverted, which is a straightforward consequence of the change in the direction of the applied field. Since, due to the applied field, $\mathbf{H}_{0}$ tends to $\left[0,0,(\alpha-1) M_{s}\right]$ at infinity, the solutions of system (22)-(23) must satisfy the boundary conditions

$$
\lim _{X \rightarrow \infty} B_{X}=0, \quad \lim _{X \rightarrow \infty} C_{X}=-\alpha .
$$

Notice that the strength of the external field does not appear in the equations, except through the boundary conditions (26).

In YIG, the normalized damping constant $\bar{\sigma}$ can be considered as a quantity of order $\varepsilon^{2}$ (see above and Refs. 21 and 22 ). In this case, $\bar{\sigma}$ must be replaced by zero in Eqs. (22) and (23) and the damping term vanishes from the asymptotic system. In fact, damping is negligible because we consider propagation times much shorter than the damping time. To estimate the latter, we assume a resonance linewidth $\Delta H$ $=0.6$ Oe for an applied field $H_{\text {ext }}=3000$ Oe, which gives $\sigma=\gamma \mu_{0} \Delta H / 2 H_{\mathrm{ext}} \simeq 1760 \mathrm{Oe}^{-1} \mathrm{~s}^{-1}$. It corresponds to $\bar{\sigma}$ $=10^{-4}$ and the damping time is then $1 /\left(M_{s} \sigma\right) \simeq 3 \times 10^{-7} \mathrm{~s}$, while the propagation time is typically $l_{0} /(c \varepsilon) \sim 3 \times 10^{-9} \mathrm{~s}$. Hence damping is neglected thereafter.

\section{Line soliton and its stability}

If transverse variations are neglected, system (22)-(23) reduces to the sine-Gordon $(\mathrm{sG})$ equation, as the system derived in the case of the in-plane field does. Hence from the kink solution of $\mathrm{sG}$, is deduced the line soliton ${ }^{20}$

$$
B=2 w \operatorname{sech} z, \quad C=w(2 \tanh z-z), \quad z=X-w T,
$$

where the soliton velocity $w$ is an arbitrary real parameter.

The stability of the line soliton (27) with respect to slow transverse perturbations is studied following the same approach as in Ref. 20. Detail of the analysis is left for further publication. It is found that the line soliton is stable if its velocity $w$ is negative and unstable for $w>0$.

Notice that $\lim _{X \rightarrow \infty} C_{X}=-w$, hence due to the boundary condition (26), the speed $w$ of the line soliton must be equal to the strength $\alpha$ of the constant field. Taking into account demagnetizing factors and relation (5), $\alpha<0$ corresponds to a magnetization in a direction opposite to the external field, which is known to be unstable. In the latter case, a transverse instability of the background was found and studied. ${ }^{22}$ It was seen that the transverse background instability could be removed by means of a narrowing of the sample. Such an instability does not occur at all here.

System (22)-(23) is solved numerically using the scheme presented in Ref. 20. First the stability of the line soliton 

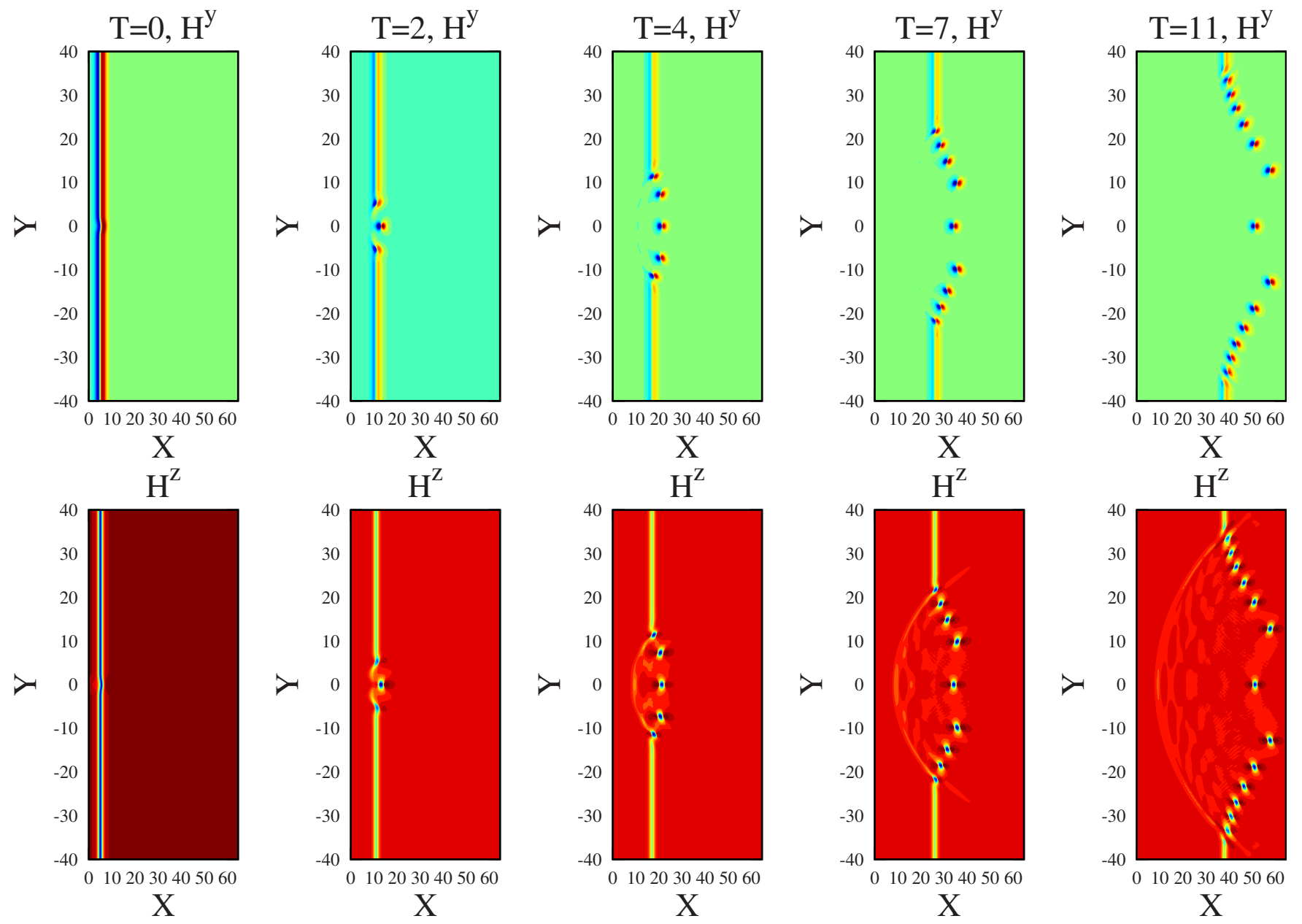

FIG. 2. (Color online) An initial unstable line soliton splits into lumps.

with negative velocity is checked numerically. However, the background is unstable since the magnetization is antiparallel to the external field. This instability expresses in the numerical results by the spontaneous arising of many line solitons. In accordance with the above analysis, the latter are robust against transverse perturbations.

The evolution of an unstable line soliton, initially perturbed, is also computed; the instability occurs and the line soliton decays into lumps. The latter are stable and propagate forward, see Fig. 2.

\section{LUMPS}

\section{A. Variational analysis}

We intend now to study these stable two-dimensional lumps by means of a variational approach. System (22)-(23) derives from the following Lagrangian density:

$$
\mathcal{L}=\frac{1}{2} C_{X} C_{T}+\frac{1}{2} B_{X} B_{T}-\frac{1}{2}\left(C_{Y}\right)^{2}-\frac{1}{2}\left(B_{Y}\right)^{2}+\frac{1}{2} C_{X} B^{2},
$$

through

$$
\frac{\delta \mathcal{L}}{\delta C}=0, \quad \frac{\delta \mathcal{L}}{\delta B}=0 .
$$

We seek for traveling solutions of Eqs. (22) and (23), including a background field $\mathbf{H}_{0}=[0,0,(\alpha-1) m]$, in accordance with the initial assumptions. Therefore we transform $B$ and $C$ must have the form $B=B(X-v T, Y)$ and $C=-\alpha X+C^{\prime}(X$ $-v T, Y)$. The equations become (dropping the primes)

$$
\begin{gathered}
-v C_{X X}=-B B_{X}+C_{Y Y}, \\
-v B_{X X}=-\alpha B+B C_{X}+B_{Y Y},
\end{gathered}
$$

and the effective Lagrangian density is

$$
\begin{aligned}
\mathcal{L}_{\text {eff }}= & \frac{-v}{2}\left(C_{X}\right)^{2}-\frac{v}{2}\left(B_{X}\right)^{2}-\frac{1}{2}\left(C_{Y}\right)^{2}-\frac{1}{2}\left(B_{Y}\right)^{2}+\frac{1}{2} C_{X} B^{2} \\
& -\frac{\alpha}{2} B^{2} .
\end{aligned}
$$

We make use of the variational approximation method with the ansatz

$$
B=p \exp \left(-\frac{X^{2}}{f^{2}}-\frac{Y^{2}}{g^{2}}\right)
$$



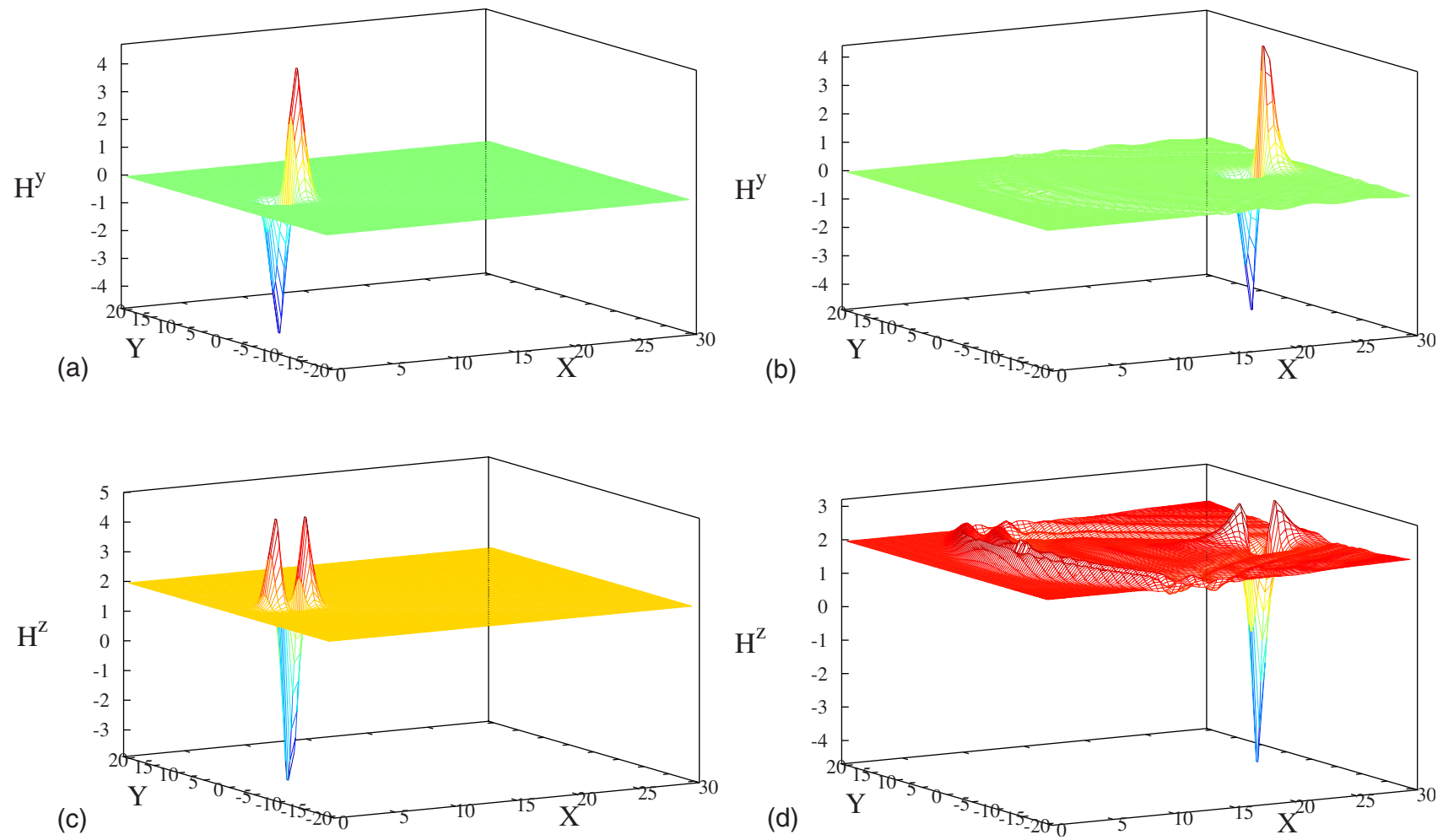

FIG. 3. (Color online) Evolution of the variational lump. (a) $H_{y}$ component, initial data given by the variational approximation. (b) $H_{y}$ after a propagation time $T=15$. (c) $H_{z}$ component, initial data. (d) $H_{z}$ at $T=15$.

$$
C=-\mu X \exp \left(-\frac{X^{2}}{f^{2}}-\frac{Y^{2}}{g^{2}}\right)
$$

The Lagrangian $L=\int_{\mathbb{R}^{2}} \mathcal{L}_{\text {eff }} d X d Y$ is computed by standard methods and then derived with respect to the dynamical variables $p, \mu, f^{2}$, and $g^{2}$, the quantities $\alpha$ and $v$ being treated as parameters. After reduction, we get the following set of equations:

$$
\begin{gathered}
9 f^{2}+9 \alpha f^{2} g^{2}+4 f^{2} g^{2} \mu+9 g^{2} v=0, \\
9 f^{2} \mu+8 g^{2} p^{2}+27 g^{2} \mu v=0, \\
9 f^{2} \mu^{2}+36 \alpha g^{2} p^{2}+16 g^{2} \mu p^{2}+27 g^{2} \mu^{2} v=0, \\
f^{4} \mu^{2}+2 f^{2} p^{2}-2 g^{2} p^{2} v=0 .
\end{gathered}
$$

It can be solved explicitly as follows: first we introduce the parameters $q$ defined by

$$
g^{2}=q f^{2},
$$

then $p$ is computed from Eq. (36), as

$$
p=\frac{-9 \mu(1+3 q v)}{8 q},
$$

then from Eq. (37) is seen that either

$$
\mu=\frac{-9 \alpha}{2},
$$

or $q=-1 /(3 v)$. It is seen that the latter condition is not compatible with the remaining equations and hence Eq. (41) holds. Equation (35) yields

$$
f^{2}=\frac{1+q v}{\alpha q},
$$

and Eq. (38) produces two solutions $q_{ \pm}=(2 \pm \sqrt{13}) /(3 v)$. Since $q$ must be positive, the solutions $q_{+}$and $q_{-}$require positive and negative values of $v$, respectively. $q=q_{-}$does not allow to obtain positive values of $p^{2}$ and $f^{2}$ simultaneously and must be rejected. Finally, we obtain one variational solution, valid if both $v$ and $\alpha$ are positive only, defined by formulas (33), (34), and (39)-(42) above and

$$
q=\frac{2+\sqrt{13}}{3 v} .
$$

We have thus obtained a two parameter family of lumps, the parameters being the applied field strength $\alpha$ and the lump velocity $v$.

\section{B. Numerical validation}

Then the variational solutions can be checked numerically. Figure 3 gives a typical example of computation, showing the evolution of an input equal to the approximate lump given by the variational analysis. Figures 3(a) and 3(c) show the two field components $H_{0}^{y}$ and $H_{0}^{z}$ of this input (the 
unit is the saturation magnetization $M_{s}$ ) while Figs. 3(b) and 3(d) show the same components after propagation and hence are close to the exact lump, as computed numerically.

The agreement is quite good. Notice, however, that the behavior at infinity does not match the Gaussian ansatz (33)(34). A fit of the numerical results shows that the decay is exponential, in both $X$ and $Y$ directions.

The above values are dimensionless, the space scales being normalized with respect to $l_{0}=c /\left(\gamma \mu_{0} M_{s}\right)$, and the field amplitudes to the saturation magnetization $M_{s}$. In physical units, the width and length of the lump are

$$
y_{l}=\frac{c}{\gamma \mu_{0} M_{s}} g, \quad x_{l}=\frac{2 \varepsilon c}{\gamma \mu_{0} M_{s}} f .
$$

Assuming values typical for YIG, as $\gamma \mu_{0}=1.759$ $\times 10^{7} \mathrm{rad} \mathrm{s}^{-1} \mathrm{Oe}^{-1}, M_{s}=1800 \mathrm{Oe}, \varepsilon_{r}=12$, and taking $\varepsilon$ $=10^{-2}$, we get for the example of Fig. $3 x_{l} \simeq 48 \mu \mathrm{m}$ and $y_{l}$ $\simeq 3 \mathrm{~mm}$. For this special example, the width of the structure is the same as found in the case of external field parallel to the sample while its length is appreciably shorter.

\section{CONCLUSION}

We have studied the nonlinear propagation of bulk polaritons in a ferromagnetic thick film with an initial saturation magnetization perpendicular to the main face of the film. By means of the short-wave approximation, we showed that the propagation is governed by a $(2+1)$-dimensional asymptotic model equation generalizing the sine-Gordon one. The model differs from the case of the in-plane applied field by the absence of transverse drift. We exhibited line solitons and analyzed their transverse stability. When they are unstable, line solitons split into lumps, which are accounted for by an approximated analytical expression with a good agreement with numerics.

\section{ACKNOWLEDGMENT}

This work was made in the framework of CNRS GDRPhoNoMi2 (Photonique Nonlinéaire et Milieux Microstructurés).
${ }^{1}$ F. G. Bass, N. N. Nasonov, and O. V. Naumenko, Sov. Phys. Tech. Phys. 33, 742 (1988).

${ }^{2}$ P. De Gasperis, R. Marcelli, and G. Miccoli, Phys. Rev. Lett. 59, 481 (1987).

${ }^{3}$ B. A. Kalinikos, N. G. Kovshikov, and A. N. Slavin, Phys. Rev. B 42, 8658 (1990).

${ }^{4}$ A. K. Zvezdin and A. F. Popkov, Sov. Phys. JETP 57, 350 (1983).

${ }^{5}$ A. N. Slavin and I. V. Rojdestvenski, IEEE Trans. Magn. 30, 37 (1994).

${ }^{6}$ H. Leblond and M. Manna, Phys. Rev. E 50, 2275 (1994).

${ }^{7}$ H. Leblond, J. Phys. A 32, 7907 (1999).

${ }^{8}$ H. Leblond, J. Phys. A 28, 3763 (1995).

${ }^{9}$ H. Leblond, J. Phys. A 36, 1855 (2003).

${ }^{10}$ H. Leblond, J. Phys. A 35, 10149 (2002).

${ }^{11}$ I. Nakata, J. Phys. Soc. Jpn. 60, 77 (1991).

${ }^{12}$ M. Daniel, V. Veerakumar, and R. Amuda, Phys. Rev. E 55, 3619 (1997)

${ }^{13}$ M. Daniel and V. Veerakumar, Phys. Lett. A 302, 77 (2002).

${ }^{14}$ M. R. F. Jensen, S. A. Feiven, T. J. Parker, and R. E. Camley, J. Phys.: Condens. Matter 9, 7233 (1997).

${ }^{15}$ V. V. Tarakanov, V. I. Khizhnyi, A. P. Korolyuk, and M. B.
Strugatsky, Physica B 284-288, 1452 (2000).

${ }^{16}$ V. H. Arakelian, L. A. Bagdassarian, and S. G. Simonian, J. Magn. Magn. Mater. 167, 149 (1997).

${ }^{17}$ R. E. Camley, J. Magn. Magn. Mater. 200, 583 (1999).

${ }^{18}$ E. S. Guimarães and E. L. Albuquerque, Solid State Commun. 122, 623 (2002).

${ }^{19}$ R. A. Kraenkel, M. A. Manna, and V. Merle, Phys. Rev. E 61, 976 (2000).

${ }^{20}$ M. Manna and H. Leblond, J. Phys. A 39, 10437 (2006).

${ }^{21}$ H. Leblond and M. Manna, Phys. Rev. Lett. 99, 064102 (2007); 100, 099902(E) (2008).

${ }^{22}$ H. Leblond and M. Manna, J. Phys. A: Math. Theor. 41, 185201 (2008).

${ }^{23}$ H. Leblond and M. Manna, Phys. Rev. B 77, 224416 (2008).

${ }^{24}$ M. A. Manna and V. Merle, Phys. Rev. E 57, 6206 (1998).

${ }^{25}$ M. A. Manna, J. Phys. A 34, 4475 (2001).

${ }^{26}$ H. Leblond, J. Phys. B 41, 043001 (2008).

${ }^{27}$ B. B. Kadomtsev and V. I. Petviashvili, Sov. Phys. Dokl. 15, 539 (1970).

${ }^{28}$ R. C. Lecraw, E. G. Spencer, and C. S. Porter, Phys. Rev. 110, 1311 (1958). 